\title{
A Self-inflicted Hot Oil Burn in a Male With a Major Psychiatric Disorder: A Case Report
}

\author{
Zahra Ismaili Shahroudi Moqaddam ${ }^{1}$, Mohsen Saberi Isfeedvajani ${ }^{*}$ \\ 'Department of Forensic Medicine, Faculty of Medicine, Iran University of Medical Sciences, Tehran, Iran \\ ${ }^{2}$ Medicine, Quran and Hadith Research Center; Department of Community Medicine, Faculty of Medicine, \\ Baqiyatallah University of Medical Sciences, Tehran, Iran
}

*Corresponding Author: Mohsen Saberi Isfeedvajani, M.D., Associate Professor, Medicine, Quran and Hadith Research Center; Department of Community Medicine, Faculty of Medicine, Baqiyatallah University of Medical Sciences, Tehran, Iran. Tel: +98-2181263617, Email: drsaberihaji@gmail.com

Received August 24, 2019; Accepted October 29, 2019; Online Published December 15, 2019

\begin{abstract}
Introduction: Suicide is a major challenge to public health worldwide, and self-inflicted burn is one of the most violent suicide methods.

Case Presentation: In this case, a self-inflicted hot oil burn in a male with schizotypal personality and major depressive disorder is reported.

Conclusion: Psychiatric disorders, marital problems, and economic poverty are mentioned as risk factors for self-inflicted burn. Thus, these risk factors should be considered in the management of self-inflicted burn patients.

Keywords: Suicide, Burn, Schizotypal Personality Disorder, Forensic Medicine, Depressive Disorder, Self-injurious Behavior
\end{abstract}

\section{Introduction}

Suicide is defined as conscious self-destruction to rid oneself of an unbearable situation. It has known psychological, social, cultural, economic, and human dimensions. Selfinflicted burn is one of most violent and painful methods of suicide $^{1}$ and the most dramatic form of suicide associated with cultural and psychiatric factors. ${ }^{2}$ Self-immolation is not a common way of suicide in developed American and European countries. ${ }^{3}$ It has been reported that 800000 die due to self-immolation worldwide, and self-inflicted burn is the third leading cause of death in people aged 15-44 years. Based on a WHO report, $80 \%$ of self-immolation occurs in low- to middle-income countries. ${ }^{4}$

Unfortunately, self-immolation is one of the most common methods of suicide in Iran, especially in the western provinces of the country. ${ }^{1,5}$ The male-to-female ratio is 1 to $9 .{ }^{1}$ In some areas of Iran, the prevalence of self-inflicted burn is more than $70 \%$. The highest rate of self-inflicted burn in males has been reported to be $47 \%$ worldwide. ${ }^{3}$ Historical evidence indicates that selfinflicted burn was endemic in India and reported to have first occurred in that country. Also, self-inflicted burn accounts for more than $40 \%$ of suicides. In Sri Lanka, selfimmolation is the most prevalent method of suicide. In Pakistan, more than $80 \%$ of patients admitted to hospital due to self-immolation were young, married, illiterate women, and the most prevalent cause of self-immolation was reported to be marital problems. ${ }^{3}$

Self-inflicted burn has several risk factors. In Iran, adjustment disorders have been reported as the most prevalent co-morbidity, while depression, insanity, alcohol use, and addiction have been reported as the most prevalent risk factors for self-immolation elsewhere. ${ }^{4}$ In Ilam province of Iran, it has been reported that female gender, marriage, mental disorders, and unemployment were risk factors for self-immolation. ${ }^{5}$ A study about burn patients referred to Shahid Motahari Burn Center confirmed that self-inflicted burn is associated with psychiatric disorders such as sleep disorders, aggression, addiction, depression, and anxiety. ${ }^{6}$ Depression as a major mental disorder was known as a risk factor for suicide in young, married women. ${ }^{7}$ Other mental disorders such as schizophrenia, affective disorders, substance abuse, ${ }^{8-12}$ economic status, and marital risk factors can also result in self-immolation. ${ }^{9}$

Based on a review of 55 studies conducted in the last 20 years, self-immolation was prevalent among males in western countries, while in eastern and Middle East countries, women preferred to use self-inflicted burn as a method of suicide. Kerosene was the common burning agent in India, eastern countries, Middle East countries, and Europe. Petrol and domestic gas was also used in Middle East countries and Europe. ${ }^{10,11}$ Although hot liquids such as boiling water, flammable chemical agents, electricity, and fire were rarely used, ${ }^{11}$ hot oil was used in

Copyright (C) 2019 The Author(s). This is an open-access article distributed under the terms of the Creative Commons Attribution License (http:// creativecommons.org/licenses/by/4.0), which permits unrestricted use, distribution, and reproduction in any medium, provided the original work is properly cited. 
$5.8 \%$ of self-immolation cases. ${ }^{12}$ Herein, we report a selfinflicted burn in a male with schizotypal personality and major depressive disorder.

\section{Case Presentation}

The patient was a single 40 -year-old man with low socioeconomic status who had lost his job and been unemployed for the past 3 years. He reported a history of hospitalization in Iran Physiatry Center (a hospital affiliated with Iran's University of Medical Sciences). He had received electroconvulsive therapy several times during his hospitalization. The patient had been diagnosed with major depressive disorder (MDD) and schizotypal personality disorder and treated with thiothixene, biperiden, and sodium valproate since his diagnosis. He reported a history of self-inflicted burn with acidic substances. The patient was admitted to Motahari Burn Center (a hospital affiliated with Iran's University of Medical Sciences) due to self-inflicted burn with hot oil. The total burn surface area (TBSA) was $15 \%$. Second- and third-degree of burns were seen on the patient's face, abdomen, and both legs. He received standard medical treatment. Psychiatric counseling was also performed during his hospitalization, and his mental health problems were reconfirmed. Finally, continued treatment with previous medications was recommended.

\section{Discussion}

Self-immolation is the most dramatic method of suicide for which risk factors including concomitant psychiatric illnesses have been established. ${ }^{2}$ This method of suicide is rarely practiced in high-income countries. Unfortunately, the increasing use of this method has been reported among women with economic and cultural poverty in India, Sri Lanka, Afghanistan, and Iran. The age of self-immolation patients ranged between 34 and 43 years in developed countries; however, most cases of self-immolation in developed countries occurred in men, ${ }^{3,9}$ who had confirmed histories of psychiatric disorders. ${ }^{3}$

In Iran, adjustment disorder is the most common psychological risk factor associated with self-immolation; however, in other countries depression, insanity, alcohol use, and addiction have been mentioned as risk factors. ${ }^{4}$ Personality disorders and low socio-economic status are other important risk factors for self-immolation. ${ }^{9}$ Several studies have reported kerosene, petrol, gasoline, and alcohol as the most common burning agents, ${ }^{10,11}$ and selfimmolation with hot liquids such as boiling water and burning chemicals (acid) has been reported in rare cases. ${ }^{11}$ Self-immolation with hot oil has been reported only in a high percentage of cases with MDD. ${ }^{12}$

Based on a study by Dobson et al, most patients with self-inflicted burn had a past psychiatric diagnosis and public mental health service contact in the month prior to the injury. However, male patients had lower rates of mental disorders and recent mental health service contact. Unfortunately, males with self-inflicted burns are more likely to die in comparison with males with non- self-inflicted burns. Dobson et al concluded that mental health professionals should screen the psychiatric history of patients with severe burn injuries and involve the acute and long-term management of patients with self-inflicted burn. ${ }^{13}$

Based on a study by Danesh et al, most self-immolation cases were women. Most of these cases had high TBSA, between $71 \%-100 \%$, with a high rate of mortality. They concluded that further studies are needed to identify preventive strategies for reducing the risk of selfimmolation, such as consultation and instructional courses during the high school years. ${ }^{14}$

Nazeer et al reported that lethality was higher in patients with self-immolation. Also, this method of suicide was a highly lethal method. Finally, impulsivity and lethality were negatively correlated. This means that if impulsivity increased, the lethality of the suicidal attempt decreased. ${ }^{15}$

\section{Conclusion}

In the present case, several risk factors including economic poverty, unemployment, MDD, schizotypal personality disorder, and a past history of self-immolation with rare burns (acid) were observed. The patient was also in the age range that has a high probability of self-immolation. Thus, these risk factors should be considered in the management of self-inflicted burn patients. Long-term mental health services should also be considered in order to prevent additional suicide attempts in these cases. A case-finding program of psychiatric disorders in the health network of the country under primary health care and the screening of self-immolation patients are recommended to be implemented in health centers in order to promote the mental health of the community.

\section{Authors' Contributions}

ZISM and MSI contributed equally to this Study.

\section{Conflict of Interest Disclosures}

The authors declare that they have no conflicts of interest.

\section{Ethical Approval}

The patient gave permission for the presentation of his case reports.

\section{References}

1. Safiri KH, Rezaeinasab Z. Qualitative study of the phenomenon of self-immolation in Ilam. Journal of Woman and Society. 2017;7(25):123-141. [Persian].

2. Zarghami M, Khalilian AR. Psychiatric and physical disorders in suicide attempted by self-burning in Mazandaran province. Hakim Research Journal. 2003;5(4):263-270. [Persian].

3. Rezaeian M. A Narrative Review on Epidemiology of SelfImmolation. Journal of Rafsanjan University of Medical Sciences. 2016;15(6):563-574. [Persian].

4. Saadati M, Azami-Aghdash S, Heydari M, Derakhshani N, Rezapour R. Self-immolation in Iran: systematic review and meta-analysis. Bull Emerg Trauma. 2019;7(1):1-8. doi:10.29252/beat-070101.

5. Yasemi MR, Yasemi M, Roghani A, Yaghoubi M, Zamani N, 
Sayehmiri K. Burning Percentage Evaluation of Cases With Suicide Attempt by Self-Burning in The Ilam Province Between 1372-1385. Journal of Ilam University of Medical Sciences. 2013;21(3):53-63. [Persian].

6. Jalali-Nadoushan A, Alavi K, Najarzadegan MR, Mehrabani P. History of mental health services use in self-immolation suicide attempters referred to motahari burn center, Tehran, Iran. Journal of Isfahan Medical School. 2016;34(374):203213. [Persian].

7. Rezaeinasab Z, Sheikhi MT, Jamilei Kohaneh Shahri F. Selfimmolation of women in Ilam city, Iran: a descriptive study. Journal of School of Public Health and Institute of Public Health Research. 2018;15(4):365-376. [Persian].

8. Atwell K, Bartley C, Cairns B, Charles A. Incidence of selfinflicted burn injury in patients with Major Psychiatric Illness. Burns. 2019;45(3):615-620. doi:10.1016/j.burns.2018.10.008.

9. Chabi F. Women self-immolation. Woman \& Culture. 2013;5(16):95-102. [Persian].

10. Laloë V. Patterns of deliberate self-burning in various parts of the world. A review. Burns. 2004;30(3):207-215. doi:10.1016/j. burns.2003.10.018.

11. Poeschla B, Combs H, Livingstone S, Romm S, Klein MB Self-immolation: socioeconomic, cultural and psychiatric patterns. Burns. 2011;37(6):1049-1057. doi:10.1016/j. burns.2011.02.011.

12. Malic CC, Karoo RO, Austin O, Phipps A. Burns inflicted by self or by others--an 11 year snapshot. Burns. 2007;33(1):92-97. doi:10.1016/j.burns.2006.04.008.

13. Dobson H, Lee S, Breadon C, Cleland H, Moncur D, Kulkarni J. How self-inflicted injury and gender impacted the outcome following a severe burn. Burns. 2019;45(3):621-626. doi:10.1016/j.burns.2018.10.021.

14. Danesh HA, Javanbakht $S$, Nourallahzadeh $M$, et al. Epidemiology and mortality of burn injuries in Eastern Iran since 2009: an analysis of 2115 cases. Int J High Risk Behav Addict. 2019;8(1):e66232. doi:10.5812/ijhrba.66232.

15. Nazeer N, Tharayil HM, Vidyadharan V. A Comparative study of impulsivity, lethality and intent among patients attempting suicide by self-immolation and poisoning. Kerala Journal of Psychiatry. 2019;32(1):1-7. doi:10.30834/KJP.32.1.2019.165. 